\title{
Cirurgia no Aspergiloma Pulmonar: Experiência Mono-Institucional
}

\author{
Pulmonary Aspergilloma Surgery: a Mono-Institutional Experience
}

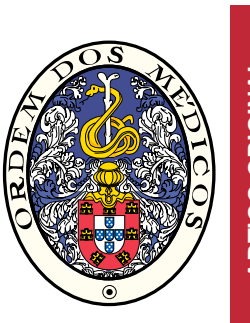

\author{
Sílvia da Silva CORREIA ${ }^{1}$, Carlos PINTO², João BERNARDO² $^{2}$
}

Acta Med Port 2014 Jul-Aug;27(4):417-421

\section{RESUMO}

Introdução: O aspergiloma ou micetoma é resultado da colonização saprofítica de uma cavidade pulmonar pelo Aspergillus. A cirurgia do aspergiloma é o único tratamento eficaz a longo prazo mas a alta incidência de complicações no intra e no pós-operatório fazem desta um tema controverso.

Objetivos: Análise da experiência de um Centro de Cirurgia Cardiotorácica nos doentes com aspergiloma pulmonar e avaliação dos fatores que influenciaram o prognóstico após a terapêutica cirúrgica.

Material e Métodos: Análise retrospetiva incluindo todos os doentes com diagnóstico de aspergiloma pulmonar sujeitos a cirurgia de ressecção durante um período de 10 anos numa instituição (Junho 2001- Junho 2011).

Resultados: Foram incluídos 22 doentes (18 homens), com idade média de 51,0 $\pm 17,4$ anos. Destes, $46 \%$ dos doentes apresentavam hábitos tabágicos, $41 \%$ hábitos alcoólicos acentuados e $50 \%$ história pessoal de tuberculose. A maioria dos doentes apresentava um aspergiloma complexo (73\%) e 17\% um aspergiloma simples. A apresentação clínica mais frequente foi a hemoptise (50\%). O procedimento cirúrgico consistiu em ressecção pulmonar atípica em $55 \%$, lobectomia em $27 \%$ e pneumectomia em $9 \%$. Dois doentes foram submetidos a toracoplastia. A mortalidade operatória foi de $5 \%$ (um doente). Em $36 \%$ dos doentes houve complicações no pós-operatório sendo as mais frequentes o pneumotórax (18\%) e o empiema (18\%). O follow-up médio foi de 52 meses (3 - 116) e a mortalidade aos cinco anos foi de $35 \%$. Destes, quatro doentes faleceram por causas não relacionadas e três eram doentes imunodeprimidos. A mortalidade nos aspergilomas simples foi de $40 \%$ e $33 \%$ nos aspergilomas complexos.

Discussão: A abordagem cirúrgica mais frequente foi a ressecção pulmonar atípica. A taxa de complicações foi sobreponível à da literatura.

Conclusão: $O$ tratamento cirúrgico do aspergiloma pulmonar apresenta uma baixa morbilidade e mortalidade pelo que permanece o tratamento de eleição nos doentes sem deterioração da função respiratória.

Palavras-chave: Aspergillus; Aspergilose Pulmonar/cirurgia.

\section{ABSTRACT}

Introduction: Pulmonary aspergiloma or mycetoma is a saprophytic colonization of a preexisting cavity by aspergilloma. Surgical resection is the only effective long-term treatment, but remains controversial because of the high rate of complications in the perioperative and postoperative time.

Objectives: Analysis of the experience of a Cardiothoracic Surgery Center for the treatment of pulmonary aspergilloma and evaluation of the prognostic factors after surgery.

Material and Methods: Retrospective analysis including all the patients with a diagnosis of pulmonary aspergilloma submitted to surgery for a 10 years period, in a single institution (June 2001-June 2011).

Results: The study included 22 patients (18 men) with a mean age of $51.0 \pm 17.4$ years. Of them, $46 \%$ were smokers, $41 \%$ were alcoholic and $50 \%$ had a previous history of tuberculosis. Most of the patients had a complex aspergilloma (73\%) and $17 \%$ a simple aspergilloma. The most common presentation was hemoptysis (50\%). The common surgical procedure performed was atypical lung resection in $55 \%$, lobectomy in $27 \%$ and pneumectomy in $9 \%$. Two patients were submitted to thoracoplasty. There was one operative death (5\%). Postoperative complications occurred in $36 \%$ and the most frequent were pneumothorax (18\%) and empyema (18\%). The mean follow-up period was 52 months $(3-116)$ and the 5 years mortality rate of $35 \%$. Of them, 4 patients died because of non-related causes and 3 were immunosuppressed patients. The mortality was $40 \%$ in the group of complex aspergilloma and $33 \%$ in the group of complex aspergilloma.

Discussion: The most common surgical procedure performed was atypical lung resection. The postoperative complications rate was similar to previous studies.

Conclusion: Surgical resection of aspergilloma presents a low morbidity and mortality. Therefore, for patients with lung function preserved, it is the preferred treatment.

Keywords: Aspergillus; Pulmonary Aspergillosis/surgery.

\section{INTRODUÇÃO}

O aspergiloma ou micetoma é resultado da colonização saprofítica de uma cavidade pulmonarpor fungos, mais frequentemente do género Aspergillus. Embora a colonização fúngica seja mais frequente nas cavidades tuberculosas, pode ocorrer também em cavidades associadas a sarcoi-

dose estadio IV, histoplasmose, nas bolhas de enfisema e nas bronquiectasias. ${ }^{1} \mathrm{O}$ espectro de patologias causadas por este fungo inclui ainda a doença invasiva (Aspergilose pulmonar invasiva) e alérgica (Aspergilose broncopulmonar alérgica). Os aspergilomas são classificados em simples ou

1. Serviço de Pneumologia. Hospital Sousa Martins. Unidade Local de Saúde. Guarda. Portugal.

2. Centro de Cirurgia Cardiotorácica. Centro Hospitalar e Universitário de Coimbra. Coimbra. Portugal.

Recebido: 25 de Setembro de 2013 - Aceite: 02 de Abril de 2014 | Copyright @ Ordem dos Médicos 2014 
complexos com base na tomografia computorizada do tórax segundo a classificação de Belcher et $\mathrm{al}^{2}$ e Daly et $\mathrm{al}^{3}{ }^{3}$ classificando-se como aspergiloma simples uma cavitação de parede fina ocorrendo num pulmão saudável enquanto o aspergiloma complexo apresenta uma parede espessa e surge num parênquima e/ou pleura com lesões sequelares. Em termos terapêuticos, a cirurgia do aspergiloma é a única opção eficaz a longo prazo, mas a alta incidência de complicações no pós-operatório, fazem desta um tema controverso. ${ }^{1,4}$ No caso da hemoptise grave, a cirurgia deve ser sempre ponderada apesar de sabermos que os doentes com mau estado geral ou má função pulmonar sejam mais propensos ao aparecimento de complicações operatórias. ${ }^{1,5}$

O objetivo deste estudo é avaliar as características clínicas dos doentes submetidos a cirurgia de resseção do aspergiloma pulmonar no nosso centro e os fatores que influenciaram o seu prognóstico.

\section{MATERIAL E MÉTODOS}

Foi realizada uma análise retrospetiva incluindo todos os doentes com diagnóstico de aspergiloma pulmonar sujeitos a cirurgia de ressecção durante um período de 10 anos num hospital universitário (Junho 2001- Junho 2011). Os dados foram colhidos através da consulta do processo clínico e incluíam variáveis clínicas, epidemiológicas, radiológicas e funcionais. Foram ainda analisados os dados relativos às complicações per e pós-operatórias e a mortalidade a curto e longo prazo (30 dias e cinco anos). Para efeitos de comparação, os doentes foram divididos em dois grupos de acordo com a classificação de Daly et al, ${ }^{3}$ Belcher e Plummer. Foi realizada análise estatística recorrendo ao software IBM SPSS Versão 17. A sobrevivência aos cinco anos foi estimada pelo método de Kaplan e Meier. Foram considerados significativos valores de $p<0,05$.

Tabela 1 - Dados epidemiológicos e antecedentes patológicos

\begin{tabular}{lcc}
\hline Dados epidemiológicos & N & $\%$ \\
\hline Tabagismo & 10 & $46 \%$ \\
Alcoolismo & 9 & $41 \%$ \\
Toxicodependência & 1 & $5 \%$ \\
Antecedentes patológicos & & \\
Tuberculose & 11 & $50 \%$ \\
Bronquiectasias & 5 & $23 \%$ \\
Imunosupressão & 4 & $18 \%$ \\
$\quad$ Linfoma & 3 & $13 \%$ \\
$\quad$ Transplante renal & 1 & $5 \%$ \\
Enfisema bolhoso & 1 & $5 \%$ \\
\hline
\end{tabular}

\section{RESULTADOS}

Foram incluídos 22 doentes: 18 homens e quatro mulheres com idade média de $51 \pm 17,4$ anos. Os dados epidemiológicos e antecedentes patológicos podem ser consultados na Tabela 1. Relativamente aos antecedentes, observou-se a existência de hábitos tabágicos em 10 doentes (46\%), alcoólicos em nove (41\%) e toxifílicos em apenas um doente. As patologias prévias incluíam tuberculose em metade dos doentes (11) e imunossupressão em quatro, incluindo um doente com transplante renal e três doentes com linfoma. Cinco doentes (23\%) tinham ainda antecedentes de bronquiectasias e um doente, de enfisema bolhoso (5\%). Em relação à queixa principal, 11 doentes $(50 \%)$ apresentavam hemoptise, cinco $(23 \%)$ tosse crónica e seis $(27 \%)$ eram assintomáticos. As provas de função respiratória demonstraram restrição em quatro doentes, obstrução em seis e alterações mistas em quatro doentes. Observou-se a existência de aspergiloma simples em seis doentes (27\%) e aspergiloma complexo em 16 (73\%). As características clínicas, funcionais e radiológicas da amostra podem ser consultadas na Tabela 2.

Relativamente à cirurgia, a abordagem cirúrgica fez-se por toracotomia póstero-lateral em todos os doentes. O procedimento consistiu em ressecção pulmonar atípica em 12 doentes (55\%), lobectomia em seis $(27 \%)$ e pneumectomia em dois (9\%). Nos doentes submetidos a lobectomia, em três foi realizada lobectomia superior direita e em três, lobectomia superior esquerda. Dois doentes foram submetidos a toracoplastia parcial de Shede com remoção do aspergiloma por apresentarem uma deterioração da função pulmonar que contraindicava uma resseção mais ampla. Dos dois doentes submetidos a toracoplastia, um faleceu por causas não relacionadas e o outro encontra-se assintomático. Na Tabela 3 podem consultar-se os procedimentos realizados, assim como a respetiva localização.

Tabela 2 - Características clínicas, funcionais e radiológicas

\begin{tabular}{lcc}
\hline Sintomas & N & $\%$ \\
\hline Hemoptise & 11 & $50 \%$ \\
Tosse crónica & 5 & $23 \%$ \\
Assintomático & 6 & $27 \%$ \\
\hline Provas funcionais & & \\
\hline Sem alterações & 8 & $37 \%$ \\
Restrição & 4 & $18 \%$ \\
Obstrução & 6 & $27 \%$ \\
Síndrome ventilatório misto & 4 & $18 \%$ \\
\hline Tipo de aspergiloma & & \\
\hline Simples & 6 & $27 \%$ \\
\hline Complexo & 16 & $73 \%$ \\
\hline
\end{tabular}


Tabela 3 - Procedimentos realizados e localização

\begin{tabular}{lcc}
\hline Procedimentos & N & $\%$ \\
\hline Ressecção pulmonar atípica & 12 & $55 \%$ \\
Lobectomia & 6 & $27 \%$ \\
$\quad$ Superior esquerda & 3 & $13,5 \%$ \\
$\quad$ Superior direita & 3 & $13,5 \%$ \\
Pneumectomia & 2 & $9 \%$ \\
Toracoplastia & 2 & $9 \%$ \\
\hline
\end{tabular}

A mortalidade operatória foi de apenas um doente, tratando-se de um doente com antecedentes de transplante renal sob terapêutica imunossupressora que desenvolveu no pós-operatório uma sepsis com ponto de partida pulmonar. Houve complicações no pós-operatório em oito doentes $(36 \%)$, incluindo pneumotórax $(n=4 ; 18 \%)$, empiema ( $n=4 ; 18 \%)$, fístula broncopleural $(n=3 ; 14 \%)$, internamento prolongado ( $>30$ dias) ( $n=3 ; 14 \%$ ) e pneumonia nosocomial $(n=2 ; 9 \%$ ). Salientamos que $75 \%$ dos doentes com complicações apresentavam antecedentes de tuberculose.

O follow-up médio foi de 52 meses (3 - 116 meses). A mortalidade aos cinco anos foi de $35 \%$. Destes, quatro doentes faleceram por causas não relacionadas e três eram doentes imunodeprimidos. A mortalidade nos aspergilomas simples foi de $40 \%$, sendo de $33 \%$ nos aspergilomas complexos. A análise da sobrevida através de curvas de Kaplan-Meier é documentada nas nas figuras 1 e 2 Observou-se recidiva do aspergiloma em apenas um doente com antecedentes de tuberculose, bronquiectasias e com hábitos alcoólicos e tabágicos mantidos, em localização diferente da zona de ressecção pulmonar atípica inicial

\section{DISCUSSÃO}

Tal como na literatura, a nossa experiência revelou um predomínio de aspergiloma nos doentes do sexo masculino e de meia-idade. O aspergiloma pulmonar surge em cavitações pré-existentes, a maioria causadas por tuberculose. Esta causa foi encontrada na nossa série em $50 \%$ dos doentes, valor superior ao geralmente encontrado na literatura. ${ }^{6,7}$ Este facto deve-se provavelmente a uma maior incidência desta doença em Portugal, comparando com outros países da Europa Ocidental e Estados Unidos. Pela mesma razão, a maioria apresentava um aspergiloma complexo, o que pode estar relacionado com os antecedentes de tuberculose, bronquiectasias ou enfisema bolhoso.

Em relação às manifestações clínicas, $27 \%$ dos doentes eram assintomáticos, percentagem superior à encontrada em outras séries. ${ }^{8}$ Este facto pode dever-se à experiência do nosso centro: o tratamento cirúrgico é o tratamento mais eficaz no aspergiloma pulmonar especialmente nos doentes sem manifestações, nos quais a morbilidade e

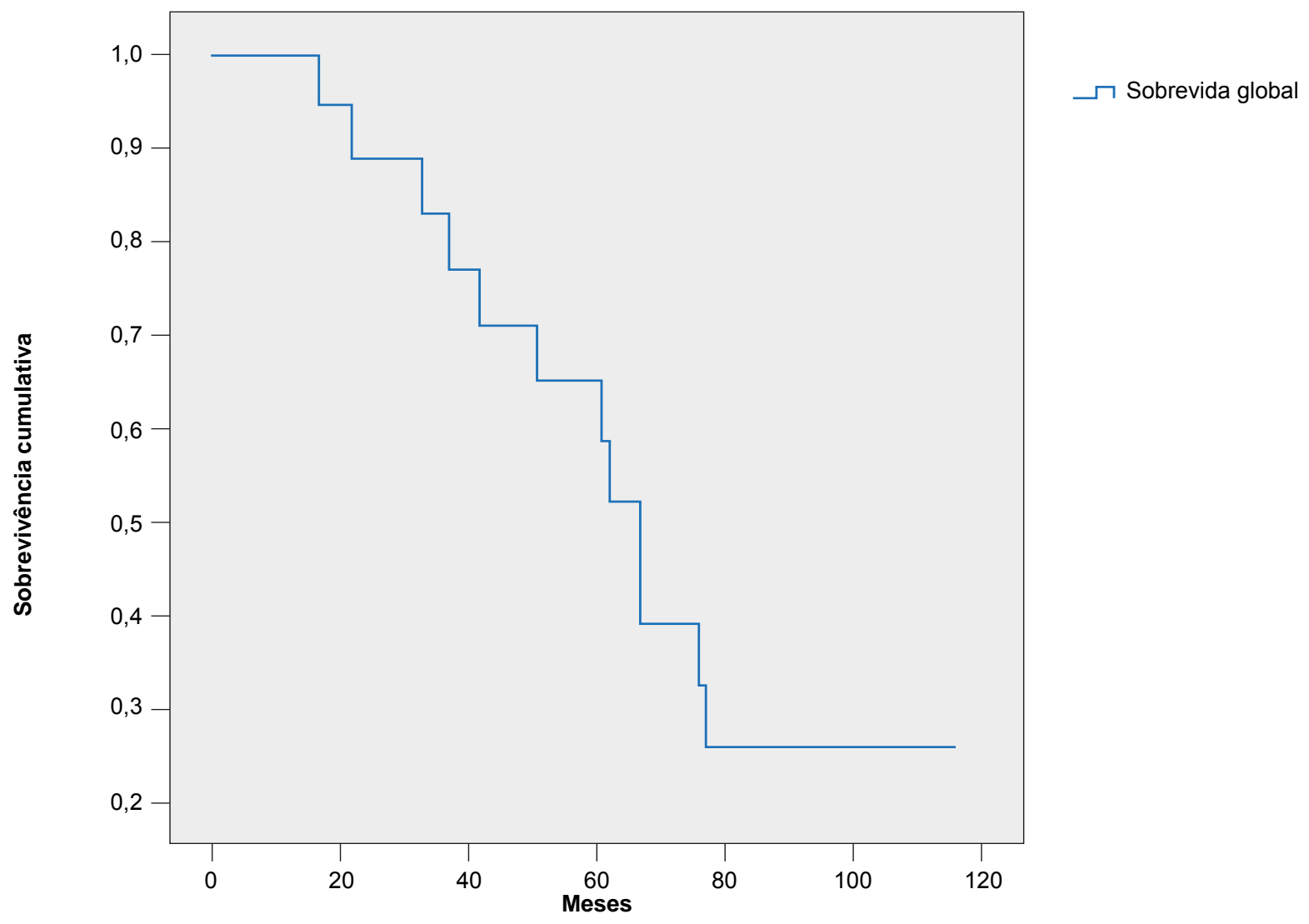

Figura 1 - Curva de sobrevida global de Kaplan-Meier 


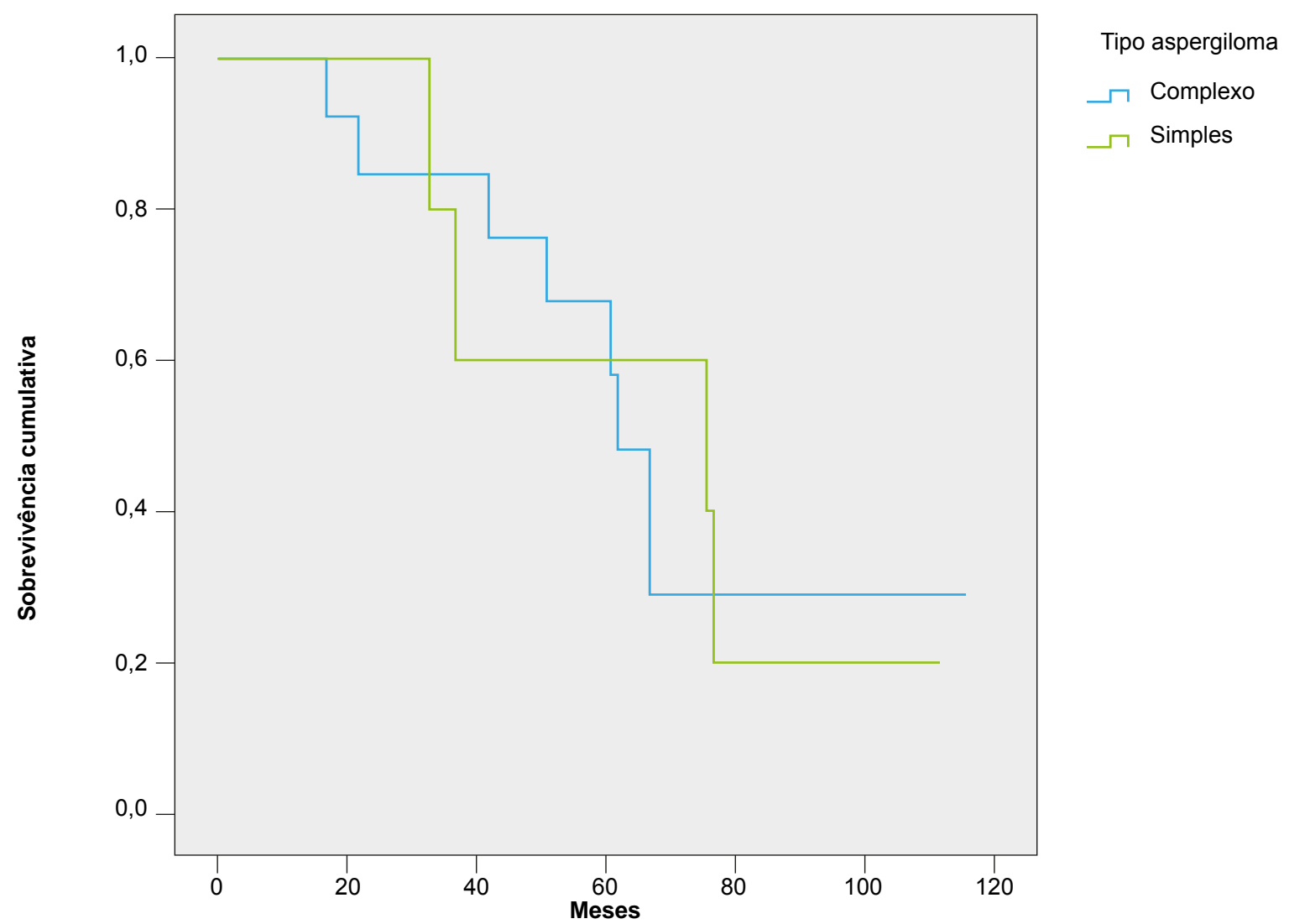

Figura 2 - Curva de sobrevida de Kaplan-Meier nos aspergilomas simples e complexos

mortalidade são baixas. De facto, estudos recentes demonstraram que a resseção cirúrgica do aspergiloma pulmonar deve ser considerada nos doentes assintomáticos que apresentem uma função respiratória razoável permitindo diminuir os sintomas e prolongar a sobrevida. ${ }^{9,10}$

Nos indivíduos sintomáticos a hemoptise foi predominante. Nestes doentes a cirurgia é o tratamento de eleição permitindo aumentar a sobrevida de $41 \%$ nos doentes sob tratamento médico para $84 \%$ nos doentes submetidos a cirurgia. ${ }^{11}$

Recentemente demonstrou-se que a resseção cirúrgica pode prevenir a recidiva da hemoptise: no estudo de Akbari et $\mathrm{al}^{7}$ e Okubo et al ${ }^{12}$ não houve recidiva da hemoptise 10 anos após a cirurgia.

Na nossa série, a resseção segmentar foi o procedimento cirúrgico mais realizado. A pneumectomia foi realizada numa minoria de doentes pelo facto deste procedimento cirúrgico acarretar um alto risco de empiema no pós-operatório, com morbilidade e mortalidade associadas. A pneumectomia é assim um procedimento que deve ser evitado no aspergiloma pulmonar. ${ }^{2,13-15}$

Dois doentes foram submetidos a toracoplastia parcial (Shede) com remoção do aspergiloma por apresentarem uma deterioração da função pulmonar, não se verificando maior taxa de complicações. Nos doentes debilitados sem condições para lobectomia ou resseção segmentar, a toracoplastia deve ser sempre equacionada.

A cirurgia do aspergiloma como foi previamente referido é referenciada na literatura como uma cirurgia com uma alta taxa de complicações. Na nossa série, apenas um doente faleceu nos 30 dias após a cirurgia tratando-se de um doente com transplante renal sob terapêutica imunossupressora que desenvolveu no pós-operatório imediato uma sepsis com ponto de partida pulmonar. Oito doentes (36\%) apresentaram complicações no pós-operatório. Destes, $75 \%$ apresentavam antecedentes de tuberculose. Esta taxa de complicações é sobreponível à observada na literatura. ${ }^{16}$

A mortalidade global nos aspergilomas simples foi de $40 \%$ e $33 \%$ nos aspergilomas complexos. Esta diferença explica-se pelo facto dos doentes com aspergiloma simples na nossa série serem doentes imunodeprimidos ou com antecedentes de tuberculose, facto que acarreta um aumento da mortalidade. O pequeno tamanho da amostra de doentes com aspergiloma simples pode também ter contribuído para este facto.

\section{CONCLUSÃO}

Os fatores relacionados com pior prognóstico foram a imunossupressão e os antecedentes de tuberculose. $O$ tratamento cirúrgico do aspergiloma pulmonar é um procedimento de risco pela presença de co-morbilidades na maioria dos doentes tornando-se fundamental uma cuidadosa avaliação do risco-benefício. No entanto, como podemos observar na nossa série, apresenta uma baixa morbilidade e mortalidade pelo que nos doentes sem deterioração da função respiratória, permanece o tratamento de eleição. 


\section{CONFLITOS DE INTERESSES}

Os autores declaram não existir conflito de interesses.

\section{FONTES DE FINANCIAMENTO}

Os autores declaram que não existiu qualquer apoio financeiro.

aspergilloma: analysis of prognosis in relation to symptoms and treatment. J Thorac Cardiovasc Surg. 2009;138:820-5.

10. Park CK, Jheon S. Results of surgical treatment for pulmonary aspergilloma. Eur J Cardiothorac Surg. 2002;21:918-23.

11. Jewkes J, Kay PH, Paneth M, Citron KM. Pulmonary aspergilloma: analysis of prognosis in relation to haemoptysis and survey of treatment Thorax. 1983;38:572-8.

12. Okubo K, Kobayashi M, Morikawa H, Hayatsu E, Ueno Y. Favorable acute and long-term outcomes after the resection of pulmonary aspergillomas. Thorac Cardiovasc Surg. 2007;55:108-11.

13. Shirakusa T, Ueda H, Saito T, Matsuba K, Kouno J, Hirota N. Surgical treatment of pulmonary aspergilloma and Aspergillus empyema. Ann Thorac Surg. 1989;48:779-82.

14. Chatzimichalis A, Massard G, Kessler R, Barsotti P, Claudon B, OjardChillet J, et al. Bronchopulmonary aspergilloma: a reappraisal. Ann Thorac Surg 1998;65:927-9.

15. Massard G, Dabbagh A, Wihlm JM, Kessler R, Barsotti P, Roeslin N, et al. Pneumonectomy for chronic infection is a high-risk procedure. Ann Thorac Surg. 1996;62:1033-8.

16. Kim YT, Kang MC, Sung SW, Kim JH. Good long-term outcomes after surgical treatment of simple and complex pulmonary aspergilloma. Ann Thorac Surg. 2005;79:94-8. 


\section{Cirurgia no Aspergiloma Pulmonar: Experiência Mono-Institucional

\author{
Acta Med Port 2014:27:417-421
}

Publicado pela Acta Médica Portuguesa, a Revista Científica da Ordem dos Médicos

Av. Almirante Gago Coutinho, 151

1749-084 Lisboa, Portugal.

Tel: +351218428 215

E-mail: submissao@actamedicaportuguesa.com

www.actamedicaportuguesa.com

ISSN:0870-399X | e-ISSN: 1646-0758

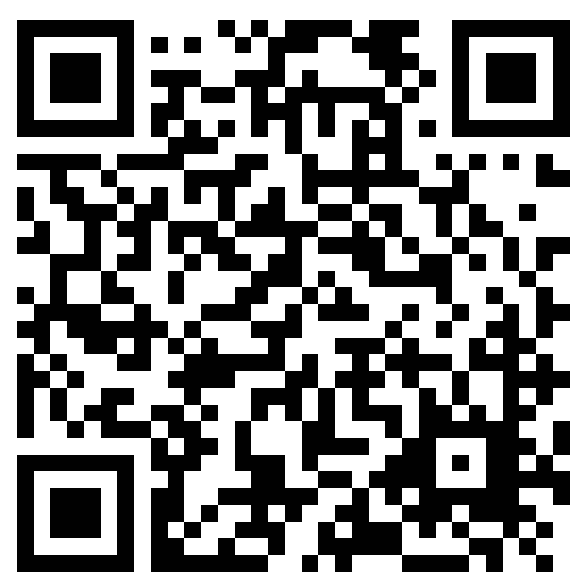

